\title{
Perfil nutricional según niveles socio-económicos y menús proporcionados en un comedor social de México
}

\author{
Nutritional profiles according to socioeconomic levels and the menu offered in a community restaurant from Mexico
}

\author{
Herenia Adilene Miramontes-Escobar' orcid.org/0000-0003-3405-5125 \\ Gladys América Prado-Guzmán'1 orcid.org/0000-0002-8869-905X \\ María de Jesús Toledo-Palomera' orcid.org/0000-0003-3779-6607 \\ Jesús Enrique Báez-García' orcid.org/0000-0001-7286-7840 \\ Sonia Guadalupe Sáyago-Ayerdi ${ }^{*}$ orcid.org/0000-0002-4430-1273
}

TTecNM-Instituto Tecnológico de Tepic, Laboratorio Integral de Investigación en Alimentos. Nayarit, México. económicos y menús proporcionados en un comedor social de México. Univ. Salud. 2020;22(3):203-212. DOI: https://doi.org/10.22267/rus.202203.192

\section{Resumen}

Introducción: México es de los principales países que enfrenta una problemática nutricional provocada por un déficit o exceso de nutrientes, que ocasiona desnutrición o sobrepeso en su población. Objetivo: Identificar los hábitos alimenticios entre tres distintos estratos sociales y compararlos con el perfil nutricional en un comedor social que brinda desayuno y comida en la Ciudad de Tepic, Nayarit. Materiales y métodos: Se realizaron encuestas en los diferentes estratos sociales que correspondieron a un recordatorio de un día de desayuno y comida con sus respectivos refrigerios y bebidas. Resultados: Se encontró consumos de proteína que excedían la ingesta recomendada diaria, bajo consumo de fibra dietética (asociado con la baja ingesta de frutas y verduras) y alta incidencia en consumo de bebidas carbonatadas. Además, la mayor ingesta calórica total fue en el estrato socioeconómico alto (atribuido a su mayor poder adquisitivo), seguido del comedor social; sin embargo, en ningún estrato, ni el comedor social se encontró una dieta balanceada. Conclusiones: El poder adquisitivo de los distintos estratos socioeconómicos no fue el principal factor que limita una adecuada alimentación, si no la falta de información y malos hábitos alimenticios de la población, debido a la pérdida de cultura alimentaria.

Palabras clave: Perfil nutricional; estratos sociales; hábitos alimenticios. (Fuente: DeCS, Bireme).

\begin{abstract}
Introduction: Mexico is among the main countries that faces nutritional problems caused by a deficit or excess of nutrients, which is causing malnutrition or overweight in its population. Objective: To identify the eating habits of people belonging to three different socioeconomic strata and compare them to nutritional profiles of a community restaurant in the City of Tepic, Nayarit. Materials and methods: The participants were surveyed about what they ate for breakfast and lunch the day before. Results: Protein consumption was found to exceed the recommended daily intake. Also, there was a low ingestion of dietary fiber (associated with low intake of fruits and vegetables) and a high incidence of carbonated drink consumption. Finally, the largest total caloric intake was recorded in people from the highest SS (attributed to their higher income), followed by those who eat at the community restaurant. However, none of the participants followed a balanced diet. Conclusions: The purchasing power of people belonging to the different socioeconomic strata is not the main factor that limits a balanced diet, since additional factors are the lack of information and poor nutritional habits of the Mexican population due to the loss of their food culture.
\end{abstract}

Keywords: Nutritional profile; social strata; eating habits. (Source: DeCS, Bireme).

\footnotetext{
*Autor de correspondencia

Sonia Guadalupe Sáyago-Ayerdi

e-mail: sonia.sayago@gmail.com
} 


\section{Introducción}

La nutrición comunitaria es un área que se encarga de la evaluación de los problemas de la población, y del diseño y ejecución de programas de intervención; tiene como objetivo mejorar el estado nutricional y de salud de los individuos y grupos poblacionales de una comunidad(1). Por tanto, entre los aspectos centrales que debe abordar, se encuentra el fomento de prácticas de alimentación saludable, que conlleva a la identificación, priorización y evaluación de problemas nutricionales de la comunidad, para implementar programas de intervención mediante distintas estrategias ${ }^{(1,2)}$.

En Latinoamérica se observa un proceso de transición nutricional, donde los hábitos de alimentación y el estado nutricional de la población, muestran que una gran parte está afectada por el déficit o el exceso de nutrientes. México es uno de los principales países que enfrenta dicha problemática, manifestada por la obesidad y desnutrición(3). Existe más de un millón de niños con desnutrición, así como millones de personas que sufren de obesidad en este país, lo cual repercute en el desarrollo de enfermedades crónicas(3). Este problema de doble carga nutricional, se ha asociado a la pérdida de cultura alimentaria, atribuida a un estilo de vida ajetreado que limita la compra, selección y preparación de alimentos; así mismo, influyen la disponibilidad y accesibilidad a alimentos con alto contenido energético y bajo costo, además de la publicidad de alimentos y bebidas procesados que acompaña el fenómeno de la globalización(4).

Los alimentos que aportan el mayor contenido energético en la dieta de los mexicanos son el maíz, seguido por azúcares simples procedentes de sacarosa y fructosa, además de otros siete productos: trigo, leche, carne de cerdo, aceite de soya, frijol, carne de aves de corral y huevo; entre todos aportan el $75,8 \%$ del suministro energético alimentario(5,6). Debido a la pérdida del poder adquisitivo y al incremento en el precio de estos productos, los sectores con mayor desigualdad social se enfrentan a la dificultad de acceder a la canasta básica alimentaria, que a su vez, genera deficiencia nutricional en esta población(3).

En diversas partes del país existen comedores comunitarios que brindan dos comidas durante el día a la población de escasos recursos, para cubrir de parcialmente la falta de alimentos(7). Es por ello que, el objetivo del presente estudio fue identificar los hábitos alimenticios entre tres distintos estratos sociales (ES) (bajo, medio y alto) con el perfil nutricional de los desayunos y comidas que se ofrecen en un Comedor Social en la Ciudad de Tepic Nayarit, en el Occidente de México.

\section{Materiales y métodos}

\section{Lugar de estudio}

En la ciudad de Tepic, Nayarit, México existen aproximadamente 351 colonias (incluyendo fraccionamientos, ejidos y rancherías aledañas), las cuales se agruparon de acuerdo a datos reportados por el Instituto Nacional de Estadística y Geografía (INEI) en el año 2000, en la Encuesta directa, Cultura política y participación electoral en elecciones locales de la ciudad de Tepic ${ }^{(8)}$. Se seleccionaron las colonias donde se pudieran encontrar ciudadanos que reciben bajos, medios y altos ingresos económicos, correspondientes al nivel socioeconómico: bajo (ESB), medio (ESM) y alto (ESA). Posteriormente, se realizó un sorteo para seleccionar una colonia por ES y aplicar encuestas correspondientes al desayuno y comida de la población.

\section{Determinación del tamaño de muestra}

El tamaño de la muestra para las encuestas realizadas, se determinó con base en el promedio estimado de habitantes por colonia, mediante el software STATISTICA versión 10 , se modificó los valores de error tipo $\alpha=0,05$ y $\beta=0,71$, y se obtuvo como resultado 64 personas por estrato socioeconómico seleccionado. La muestra corresponde a tres colonias/fraccionamientos, cada una perteneciente a un estrato.

\section{Ejecución de encuestas}

Las encuestas fueron realizadas de manera personal por estudiantes de Maestría en Ciencias en Alimentos del Tecnológico Nacional de México/ITTepic a 192 personas responsables de proveer el alimento en su hogar (personas de 20 años en adelante). Los cuestionarios correspondieron a un recordatorio de un día, donde se describió específicamente los alimentos consumidos durante el desayuno y comida, incluyendo refrigerios y bebidas. La cena fue omitida debido a que en el único comedor social de Tepic perteneciente al Sistema Nacional para el Desarrollo Integral de la Familia (DIF), con el que se realizó el comparativo, no la incluía en su menú. 
Se determinó la ingesta calórica de los encuestados para los tres ES y para cada menú proporcionado por el comedor social de apoyo (CSA). Se utilizó la Guía Alimentaria del Sistema Mexicano de Alimentos Equivalentes ${ }^{(9)}$, así como el programa Smaebook. Los resultados de las encuestas fueron comparados entre sí y a su vez con un menú semanal del CSA para personas de bajos recursos.

\section{Análisis estadístico}

Los datos obtenidos fueron analizados utilizando el software STATISTICA versión 10 (StatSoft, USA) mediante un ANOVA de una vía, para la determinación de las diferencias significativas entre los tres diferentes ES, tomando en cuenta el promedio para cada comida. Se utilizó la prueba de $t$-Student, para realizar las comparaciones de los menús del comedor social DIF con cada uno de los estratos (CSA con ESA, CSA con ESM, CSA con ESB).

\section{Consideraciones éticas}

Este tipo de investigación de campo, según las reglamentaciones mexicanas no requieren aval del comité de ética, ya que la información fue confidencial, no se tomó ningún dato personal de los participantes, ni se tomaron muestras de las mismas. Además para su desarrollo se tuvo en cuenta todas las recomendaciones consagradas en la declaración de Helsinki de 1975 modificada en 2004.

\section{Resultados}

Se presenta el perfil nutricional obtenido a partir del consumo de alimentos reportados en las encuestas, el cual muestra el porcentaje de consumo de carbohidratos, proteínas y lípidos para cada ES y el CSA del DIF, así como también la cantidad de calorías totales consumidas en un recordatorio de un día, además de los grupos de alimentos más consumidos $\mathrm{y}$ la ingesta promedio de refrescos.

\section{Frecuencia de alimentos más consumidos en los tres ES encuestados}

En relación al consumo de carbohidratos en los estratos y el comedor social (Tabla 1) se encontró diferencias significativas, siendo el ESB el mayor consumidor de éstos $(59,81 \pm 15,31 \%)$ y el ESA el de menor consumo $(37,49 \pm 13,61 \%)$. Asimismo, el ESB presenta valores similares a los del CSA $(58,14 \%)$.
Referente al consumo de proteínas se puede apreciar que el ESM presenta diferencias estadísticas significativas con respecto al ESB y ESA, siendo el mayor consumidor de proteínas $(24,24 \pm 9,04 \%)$, a su vez, dicho estrato no presenta diferencias significativas con el CSA analizado mediante la prueba t-Student, lo cual indica que el CSA se encuentra como uno de los mayores consumidores de proteínas después del ESM.

En relación con la ingesta de lípidos, existen diferencias estadísticamente significativas entre los tres ES, evidenciando a ESA como el mayor consumidor $(43,55 \pm 14,51 \%)$ y al ESB como el estrato que menor porcentaje de grasas consume $(23,51 \pm 14,46 \%)$. Los tres ES presentan diferencias significativas con respecto a los valores proporcionados por el CSA, siendo éste el de menor ingesta de grasas de todos los grupos comparados en el presente estudio.

Por otra parte, se puede observar que el consumo de carbohidratos el ESB y el CSA se encontraron dentro del rango de ingesta recomendada, mientras que el ESM y ESA no alcanzaron el porcentaje mínimo recomendado. El consumo de proteínas se muestra por encima de los valores recomendados en el CSA y en los tres ES. La ingesta de lípidos el ESB, ESM y el CSA, se mantiene dentro del rango recomendado de consumo, a excepción del ESA que excede el 13\% del máximo porcentaje sugerido(10).

La cantidad de calorías totales consumidas presenta diferencias significativas entre los diferentes ES y el CSA, siendo el ESA el que presenta la mayor ingesta calórica $(1532,02 \pm 196,68 \mathrm{Kcal})$ y el ESM el de menor ingesta calórica $(550,98 \pm 238,52 \mathrm{Kcal})$, esto evidencia la diferencia en la ingesta calórica total con relación al nivel socioeconómico.

Los resultados del análisis de frecuencia de los alimentos mayormente consumidos por los distintos ES muestran una amplia variabilidad para algunos grupos de alimentos (Figura 1).

Los vegetales y alimentos de origen animal son los grupos de alimentos con mayor frecuencia de consumo de acuerdo a lo que se reporta en ESM y ESA.

En relación al consumo de alimentos durante la comida, se pudo observar mayor tendencia en el consumo de verduras, productos de origen animal y cereales en cada estrato social, lo cual representa el 
plato promedio de cualquier mexicano, constituido por una proteína, cereal (arroz o tortilla) y algún acompañamiento como salsa mexicana, usualmente hecha a base de verduras frescas.

Tabla 1. Porcentaje de ingesta de nutrientes en los estratos y en el comedor social de apoyo en un recordatorio del desayuno y comida

\begin{tabular}{ccccc}
\hline \multirow{2}{*}{ Estrato social } & \multicolumn{4}{c}{ Porcentaje de ingesta de nutrientes } \\
\cline { 2 - 5 } & Carbohidratos & Proteína & Lípidos & Calorías totales (Kg/g) \\
\hline ESB & $59,8 \pm 15,30^{\mathrm{C}}$ & $16,7 \pm 6,50^{\mathrm{A}^{*}}$ & $23,51 \pm 14,46^{\mathrm{A}^{*}}$ & $666,38 \pm 268,82^{\mathrm{A}}$ \\
ESM & $45,8 \pm 15,34^{\mathrm{B}^{*}}$ & $24,2 \pm 9,00^{\mathrm{B}}$ & $29,94 \pm 11,94 \mathrm{~B}^{*}$ & $550,98 \pm 238,52^{\mathrm{A}^{*}}$ \\
ESA & $37,5 \pm 13,60^{\mathrm{A}^{*}}$ & $19,0 \pm 7,40^{\mathrm{A}^{*}}$ & $43,55 \pm 14,51^{\mathrm{C}^{*}}$ & $1532,02 \pm 196,68^{\mathrm{B}^{*}}$ \\
CSA & 58,10 & 22,60 & 19,26 & 727,54 \\
\hline
\end{tabular}

Los valores se expresan como el promedio \pm desviación estándar. Letras expresan diferencias significativas $(p<0,05)$ entre filas determinadas mediante una prueba LSD de Fischer. Los asteriscos $\left({ }^{*}\right)$ expresan diferencias significativas determinadas mediante una prueba t-Student respecto a los valores obtenidos para el CSA y cada uno de los tres diferentes ES.

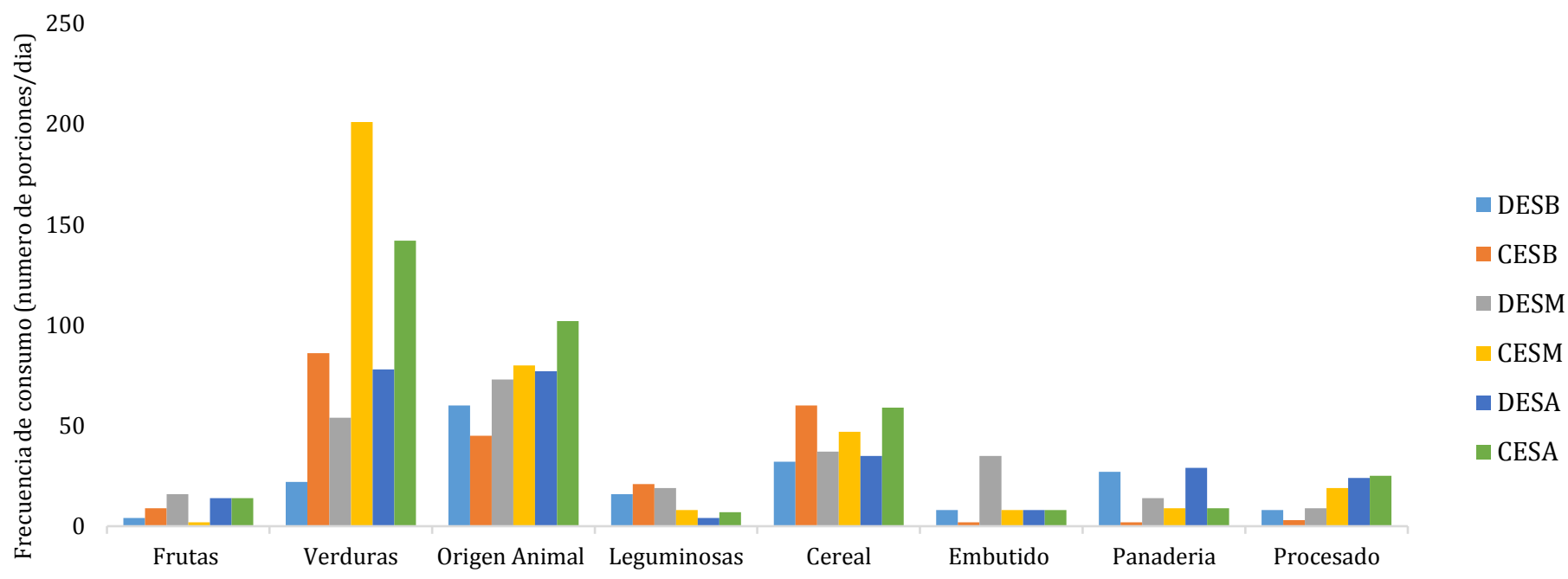

Figura 1. Grupos de alimentos más consumidos por cada estrato social al desayuno. Desayuno estrato social bajo (DESB); comida estrato social bajo (CESB); desayuno estrato social medio (CESM); comida estrato social medio (CESM); desayuno estrato social alto (DESA); comida estrato social alto (CESA)

En un análisis general de los alimentos más consumidos por el ESB se encontró que en su dieta predomina el fríjol, tortilla, leche y productos de panadería; mientras que en ESM primaron la leche y sus derivados, huevo y embutidos; sin embargo, para ESA se resalta el alto consumo de carne de res y pollo. Finalmente, con relación a los comestibles proporcionados por los CSA, al igual que los estratos sociales bajo y medio, prevalece el consumo de frijol, huevo y tortilla. En general, el frijol y el maíz representan la base de la alimentación de los encuestados respecto a la comparativa de los tres estratos sociales analizados.

La ingesta promedio de refrescos (Figura 2) para los ES (el CSA reporta la ingesta de únicamente agua purificada) revela que el ESA es el mayor consumidor de bebidas gaseosas (40\%), seguido del ESB (35\%) y el ESM (25\%). Es importante mencionar, que un alto porcentaje en la población (datos no mostrados) reportaron alto consumo de bebidas a base de frutas o plantas como la jamaica. 


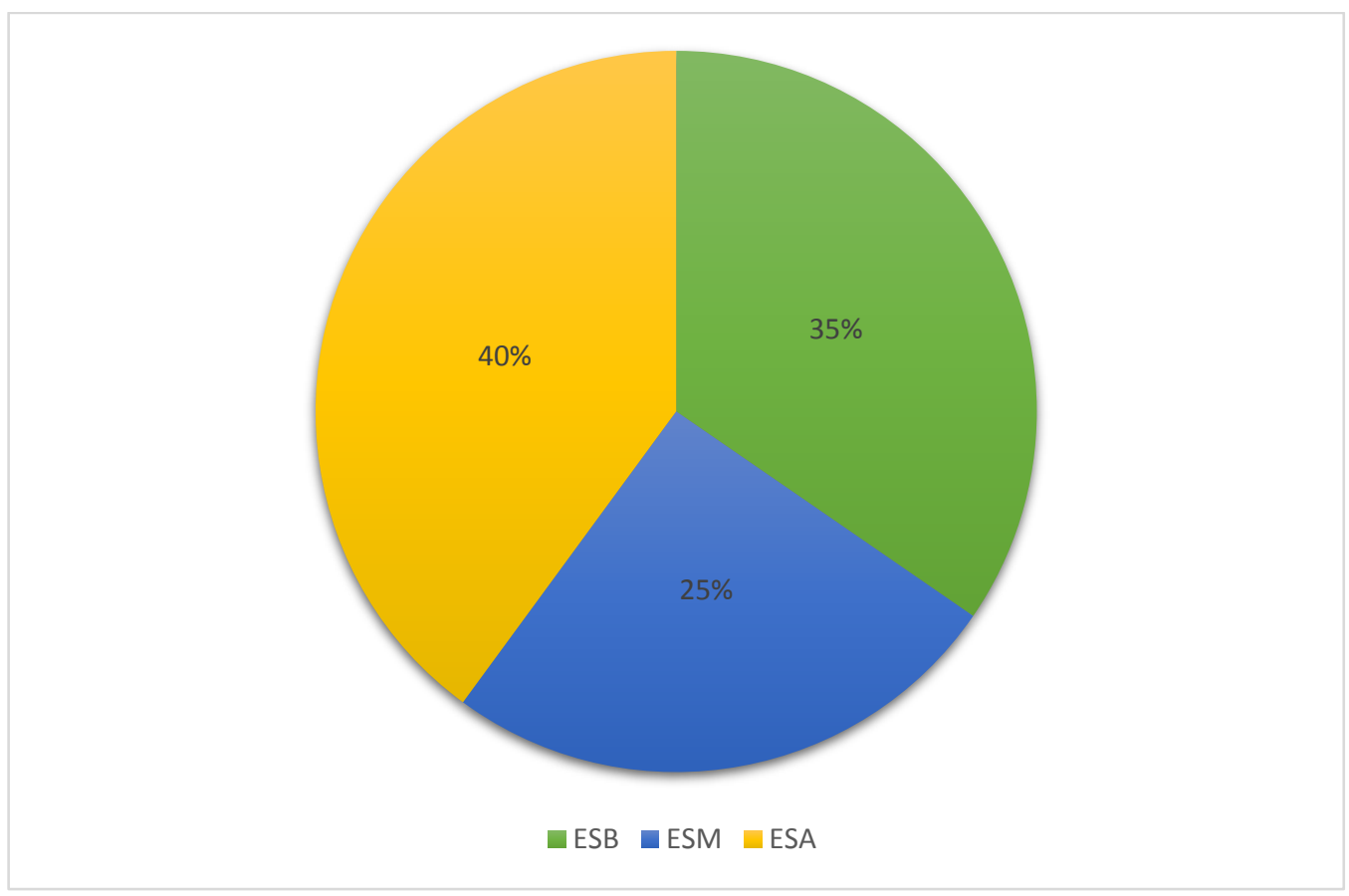

Figura 2. Ingesta promedio de refrescos incluida en la dieta alimenticia de los 3 estratos estudiados ESB: Estrato social bajo. ESM: Estrato social medio. ESA: Estrato social alto

\section{Discusión}

Una de las principales causas de la alta ingesta calórica, se debe a que en gran medida se ha sustituido el consumo de cereales de grano entero, leguminosas y verduras, por el consumo de alimentos elaborados con harinas refinadas, altos en azúcares simples y ricos en grasa hidrogenada, que se suman a la falta de actividad física y el acelerado ritmo de vida, contribuyendo al desarrollo de enfermedades crónicas no transmisibles como la hipertensión, diabetes u obesidad(11). Según la Organización para la Cooperación y Desarrollo Económicos, la obesidad ubica a México en los primeros lugares de prevalencia a nivel mundial(12), esta patología se caracteriza por un desorden metabólico, con ruptura en la homeostasis de la dinámica energética, la cual depende de la ingesta de una dieta equilibrada que conlleva a la interacción con otros factores como la susceptibilidad genética, estilo de vida, entre

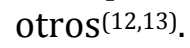

Se sabe que cada nutriente tiene sus propias funciones en el organismo, y el exceso o deficiencia de uno o más nutrientes, puede desencadenar diversas afecciones como la obesidad(14). Es importante recalcar que en este estudio no se consideró la ingesta calórica durante la cena; sin embargo, se realizó una correlación respecto a las calorías de la cena básica de un mexicano de clase media, la cual incluye el consumo de frijol, tortilla y/o pan dulce y café, obteniendo un aproximado de entre 350 y $400 \mathrm{Kcal}$, lo cual representa entre el 17,5 y $20 \%$ de las calorías totales, considerando que una dieta promedio puede ser de $2000 \mathrm{Kcal} /$ día.

La OMS(10) recomienda para la población mexicana, una ingesta de $55 \%$ a $75 \%$ de carbohidratos, $10 \%$ a $15 \%$ de proteínas y $15 \%$ a $30 \%$ de lípidos, para que la dieta pueda ser considerada como balanceada.

Los azúcares simples que se encuentran en los alimentos procesados y refinados pueden presentar efectos negativos para la salud cuando son consumidos en cantidades mayores a $30 \mathrm{~g} /$ día $^{(15,16)}$. Estudios como el de Neacsu(17) y Seidelmann(18), sustentan que cuanto más refinados sean los carbohidratos, más rápido se liberará la glucosa a la sangre y esto puede causar respuestas glucémicas elevadas y caídas abruptas en el nivel de glucosa en sangre, dando como consecuencia niveles de energía menos estables en el cuerpo. Por otro lado, los carbohidratos complejos que pueden ser diferentes tipos de almidón, como los almidones de digestión lenta, que se encuentran en verduras y frutas frescas, granos enteros y fríjoles; proporcionan una liberación 
gradual de la glucosa en sangre, por cuanto contribuyen a una glucemia posprandial más estable, manteniendo así, los niveles de saciedad por más tiempo, y como consecuencia un nivel energético más estable(19,20). Según Augustin et al.(21), debido al consumo excesivo de alimentos, hay una mayor generación de radicales libres, este estrés oxidativo puede ser el mecanismo latente de resistencia a la insulina, la diabetes y las enfermedades cardiovasculares(21).

El alto consumo de proteína puede generar un porcentaje elevado de las mismas en el organismo, haciendo que esta no pueda ser digerida y por ende, pasa al colon como proteína resistente(22). Bajo estas circunstancias la ingesta excesiva de proteína puede producir el desarrollo de padecimientos como colitis ulcerosa, inclusive cáncer colorrectal (CCR). Esto se debe a que la fermentación colónica de las proteínas se considera perjudicial para la salud del huésped, debido a que, al agotarse los carbohidratos, existe una alta disponibilidad de proteína que junto con la microbiota colónica, originan la producción de metabolitos potencialmente tóxicos como amoniaco, aminas y sulfuros. El CCR y colitis ulcerosa aparecen principalmente en el colon distal, el cual es el lugar donde ocurre la fermentación de proteínas(23,24). Este aumento en el consumo de proteínas por parte de los mexicanos puede explicarse gracias a proyecciones hechas por la FAO en el año 2005(25), las cuales expresaban que el consumo de carnes de ganado bovino y cerdo aumentaría en los siguientes años, siguiendo la tendencia mundial, en comparación con otros años, donde la cantidad de consumo era menor. Asimismo, el consumo de aves de corral ha tenido un aumento significativo desde principios del 2000, pues según encuestas, principalmente la carne de pollo es de las más consumidas por las familias mexicanas(24).

En la actualidad, el sobrepeso y la obesidad se han convertido en uno de los principales factores de riesgo a la salud en México, que también se refleja en el factor estético, con el agravante de que en la mayoría de los casos, por falta de cultura alimentaria y el afán de perder peso en corto tiempo, las estrategias seguidas por la población no siempre son las adecuadas o no cuentan con un sustento científico(26,27). Según Garza y Hernández(28), desafortunadamente en México han surgido hábitos alimentarios equivocados para perder peso en poco tiempo o "llevar una alimentación que no permita subir de peso", que finalmente representan un riesgo a la salud.

Dicho comportamiento social, pudiera verse reflejado en el presente trabajo, si bien se observa una tendencia muy marcada de que a menor poder adquisitivo mayor es la ingesta de carbohidratos debido a la fácil accesibilidad de estos productos en comparación con otro tipo de alimentos, los ES que se encontraron dentro del rango de ingesta de carbohidratos (ESB y CSA) alcanzaron porcentajes cercanos al mínimo recomendado. Estos datos demuestran que la población va siguiendo patrones o conductas alimenticias equivocadas que día con día se propagan, en este caso se presentan bajos consumos de carbohidratos que conllevan a diversas afecciones o descompensaciones en el organismo(28).

Algunas de las prácticas que han sido ampliamente difundidas por medios de comunicación colectiva son las dietas muy restrictivas y muy bajas en calorías (<800 kcal/día), bajos o nulos consumos de carbohidratos y la sustitución de los mismos por elevadas cantidades de proteínas(29,30). Dietas bajas en carbohidratos que son utilizadas en ocasiones en el tratamiento del sobrepeso, ocasionan una disminución en la cantidad de glucosa disponible que obliga al organismo a utilizar otros nutrientes (proteínas, ácidos grasos) como fuente de energía $(17,29,30)$. Finalmente, este tipo de dietas obliga al organismo a compensar la falta de energía con un aumento de la destrucción de sus propias proteínas corporales, para así conseguir una fuente alternativa de energía. Esta situación provoca pérdida de masa muscular y formación de cuerpos cetónicos, que resultan peligrosos para el organismo cuando su presencia se prolonga en el tiempo ${ }^{(31)}$. Al restringir la ingesta de carbohidratos en mayor o menor medida, se consumen proporcionalmente más proteínas y más grasas que, en exceso, pueden provocar un mal funcionamiento del organismo. Además, la exclusión total o parcial de determinados alimentos ricos en carbohidratos hace que se pierdan otros nutrientes asociados a estos, como la fibra dietética de los cereales integrales y las frutas, y muchas de las

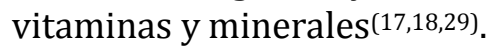

Algunos estudios de seguimiento han mostrado que el consumo prolongado de dietas pobres en carbohidratos y ricas en proteínas, se asocia con un incremento en la mortalidad, además de generar situaciones de desnutrición o déficit de diferentes 
tipos de micronutrientes y aumentar el riesgo cardiovascular(17,29).

\section{Frecuencia de alimentos más consumidos en los tres ES encuestados}

Durante el desayuno, el consumo de productos de origen animal como la leche y sus derivados, huevo y carne fueron los más consumidos, encontrándose que a medida que el estrato social tiene una mayor economía, existe un aumento en el consumo de carne(24).

Los productos de menor consumo, fueron frutas para el ESB y productos procesados para ESM y el grupo de leguminosas para ESA. El grado de consumo de los alimentos depende de cada estrato social, es decir, el ESA tiende a consumos mayores y de hasta 1,5 porciones por producto de origen animal por persona, mientras que en el ESB el consumo es de 0,7 porciones, es decir que no alcanza una sola porción por persona durante la comida, esto también comparado con el ESM con 1,25 porciones de producto por persona. Lo anterior refleja que el poder de adquisición entre estratos sociales influye en la cantidad y frecuencia en que los encuestados reportaron el consumo de dichos alimentos(24).

El consumo de lácteos al desayuno y comida fue marcado en los tres estratos sociales. En México, se estima que el 10,7\% del gasto de consumo masivo se destina a productos lácteos(32), esto supone un promedio de $217 \mathrm{~L}$ al año/familia. De esta manera, como lo afirma Martínez(24), el consumo de alimentos de cada ES está dado por la capacidad adquisitiva, que en consecuencia, si se considera el crecimiento de los precios en productos básicos como el maíz, tortilla, huevo, carne de res, pollo y cerdo, el consumo sería aún más desigual, debido a que los sectores con menor recurso social y económico son los que más padecen por el incremento de precio en la canasta básica alimentaria, dificultándose el abasto de dichos productos, generando una deficiencia nutricional.

\section{Ingesta de fibra dietética en los ES y CSA encuestados}

El análisis de datos correspondiente al presente estudio no reveló diferencia estadística significativa entre el consumo de fibra dietética (FD) en los tres ES, sin embargo, las personas del ESA consumen $30 \%$ más fibra (5,51 g/día) que el ESM (3,83 g/día) y el $17 \%$ más $(4,56$ g/día) que el ESB. Por su parte, se encontró que el consumo de fibra en los CSA es escasamente superior (5,48 g/día) al obtenido en el
ESA (5,46 g/día), es decir, la ingesta de fibra dietética recomendada en los menús analizados, está por debajo de la recomendada (25-30 g/día) ${ }^{(33,34,35) .}$

Así mismo, se realizó la comparación de ingesta de FD por comidas (desayuno y comida del "medio día"), en la cual no se encontró diferencias significativas, sin embargo, los datos permitieron observar que el mayor consumo de fibra se reporta durante la comida del "medio día" (4,69 g/día) y el menor consumo en el desayuno (4,57 g/día). La deficiencia de FD en la dieta de las personas encuestadas puede atribuirse principalmente a la baja ingesta de frutas y verduras, que probablemente se presenta por desconocimiento de la población en general y de las instituciones gubernamentales en los CSA(10,36).

Así mismo, las cifras recabadas por la Secretaría de Salud a través de la "Encuesta Nacional de Salud y Nutrición de Medio Camino 2016 (ENSANUT MC 2016)", exhiben que el consumo de FD en los ES del Estado de Nayarit, se encentra muy por debajo de la ingesta recomendada, con valores inferiores a los reportados en el último estudio nacional(37). Esta encuesta indicó que los adultos mexicanos consumían entre 16 y $18 \mathrm{~g}$ de FD al día, cifras que cubren tan solo la mitad de la recomendada, sin embargo, el presente estudio realizado en el Estado de Nayarit, representa una situación de mayor preocupación, ya que la ingesta reportada no alcanza a cubrir ni siquiera un $20 \%$ de la FD recomendada para la población mexicana(37).

La deficiencia en el consumo de FD representa un potencial problema nutricional, ya que ésta se reconoce como un elemento importante para la nutrición sana, dado que una ingesta rica en fibra acompañada de un estilo de vida saludable previene enfermedades en el colon (38,39). Un estudio realizado en Cuba reveló que entre 1960 a 1989 hubo un incremento en el consumo de alimentos ricos en grasas saturadas y carbohidratos refinados, pero muy pobres en contenido de FD, lo cual se asoció con un incremento de enfermedades crónico degenerativas, casos de hipertensión arterial (HTA) y diabetes mellitus (DM) ${ }^{(38,40)}$. De igual manera, estudios como el de Ibarra ${ }^{(40)}$ en el año 2016, muestran que en México ha habido un incremento exponencial en enfermedades crónico degenerativas durante los últimos años, lo cual es altamente asociado al bajo consumo de FD. 


\section{Ingesta de refrescos en la dieta alimenticia de los tres estratos estudiados}

El ESA reporta el mayor consumo de refresco al día con un consumo promedio de 595,71 mL ingeridos durante el desayuno y la comida, seguido por el ESB con un consumo de 516,20 mL durante la comida, por otro lado, el ESM fue el que menor consumo de refresco presenta ya que solo consumió $380,5 \mathrm{~mL}$ de este producto durante comida. En estudio de la Universidad de Yale en Estados Unidos, fue reportado, el mexicano promedio bebe $163 \mathrm{~L}$ al año, lo que representa medio litro al día, entre los datos más relevantes de la investigación, se encontró que el consumo de bebidas es la principal fuente de calorías de la dieta, ya que en promedio se ingiere $50 \mathrm{~g}$ de azúcar equivalentes a 200 kilocalorías extras(41). Lo que equivale a beber $466 \mathrm{~mL}$ diarios de refresco, equivalentes a consumir 1,3 latas de $355 \mathrm{~mL} /$ día, al año, lo cual equivaldría consumir 459 latas (42,43). Es posible observar que el consumo en los tres estratos estudiados sobrepasa las cifras reportadas por OMS para nivel máximo diario de consumo, el cual no debe superar el $10 \%$ de calorías diarias(44).

Según datos del Instituto Nacional de Estadística Geografía e Informática (INEGI), el 60\% de los hogares mexicanos se consume refresco, es decir, que en cada uno de los 17.7 millones de hogares, el $60 \%$ de la población es obesa(45). El consumo excesivo de bebidas gaseosas o azucaradas como el refresco tiene efectos negativos a la salud, ocasionando graves afectaciones a la salud como diabetes mellitus $(46,47,48)$, síndrome metabólico, dislipidemia, enfermedad cardiovascular $(48,49,50,51,52)$, osteoporosis( ${ }^{(53)}$ y cáncer ${ }^{(54,}$ 55,56,57). En el CSA del DIF no se registra la ingesta de refrescos o alguna otra bebida diferente al agua fresca de frutas en los menús proporcionados por mes, esto se debe a que promueven el consumo de bebidas saludables que contengan un aporte nutricional balanceado.

\section{Consumo de frutas incluido en la dieta alimenticia de los 3 estratos estudiados y del comedor de apoyo (DIF)}

El consumo de frutas y verduras es considerado un factor protector ante el riesgo de enfermedades crónico-degenerativas que actualmente generan más del 63\% de la mortalidad en el mundo, por ello la OMS recomienda un consumo mínimo de $400 \mathrm{~g}$ de frutas y verduras, ya que aportan suficiente FD, así como una gran cantidad de nutrientes esenciales y no esenciales, sin incrementar demasiado las calorías en la dieta(38,40,58). En la presente investigación los CSA no registraron ninguna ingesta de fruta en los menús proporcionados, el ESB indicó que solamente el 3\% de los encuestados consumieron frutas (plátano y manzana), el 20\% perteneciente al ESM consumió plátano, manzana y fresa, mientras que, para ESA se encontró que el $34 \%$ de la población consume fruta de diversas variedades tanto como frutos de temporada y otros más con un consumo mayor a los demás estratos en un día.

Si bien un consumo de frutas y verduras adecuado podría ayudar a prevenir tanto la obesidad como la incidencia de enfermedades crónicodegenerativas(59), en México la evidencia obtenida a partir de esta investigación mostró que menos del $20 \%$ de la población consume frutas, evidenciando la necesidad de desarrollar estrategias que promuevan la ingesta de estos alimentos. Esta tendencia de bajo consumo de frutas y verduras en los en los ESB y ESM, puede atribuirse a su alto costo en comparación a leguminosas como el frijol, menos consumidas por el ESA. Por otra parte, se podría considerar que en este último estrato se observó un alto consumo de productos procesados debido a que tienen mayor posibilidad de comer fuera de casa, ya que la información proporcionada por los encuestados en el ESA mostró una mayor tendencia a consumo de comida rápida(24).

\section{Conclusiones}

Los resultados obtenidos en el presente estudio revelan una situación muy alarmante respecto a la alimentación de las personas encuestadas, pues existe una extremada deficiencia en el consumo de fibra dietética (relacionada a su vez con la escasa ingesta de frutas y verduras), el consumo de proteínas es elevado y la ingesta de carbohidratos complejos es baja. Además, es importante destacar el elevado consumo de refrescos que continua en aumento en la población mexicana. La comparación de los diferentes estratos sociales revela que el factor económico no es la principal causa de la alimentación desbalanceada, dado que en los menús proporcionados por el CSA del (DIF) tampoco se encontraron resultados favorables.

La mayor limitante para que llevar una dieta balanceada es la falta de información y malos hábitos alimenticios de la población mexicana, debido a la pérdida de cultura alimentaria atribuida a un estilo de vida ajetreado que limita la compra, selección y preparación de alimentos nutritivos. 


\section{Referencias}

1. Aranceta J, Ortega RM, Salvador G, Arija V, Bautista I, Puig M. Nutrición comunitaría. Rev Esp Nutr Cominutaria. 2006;50 (4):39-45. http://bvsper.paho.org/texcom/nutricion/6NUTRICIONc.pdf

2. Holston D, Cater M, Tuuri G, O’Neil C. Exploring the Rural Nutrition Enviroment: A case Study from Lousiana. Curr Dev Nutr. 2018. 2(11).

3. De Mendoza MM. Desafíos de la nutrición comunitaria en Latinoamérica. Rev Esp Nutr Comunitaria. 2010;16:41-44. https://pesquisa.bvsalud.org/portal/resource/pt/ibc80451

4. Rivera-Domarco JA, Hernández-Ávila M, Aguilar-Salinas CA, Vadilla-Ortega F, Murayama-Rendón C. Obesidad en México: recomendaciones para una política de estado. /Libros UNAM/. 2015

5. Ortiz-Andrellucchi A, Serra-Majem L. Public Health Nutrition, Preventive Nutrition, Community Nutrition. Ref Mod Food Science. 2019 (2):214-222.

6. Soria G, Palacio V. El escenario actual de la alimentación en México. Textos \& Contextos (Porto Alegre). 2014;13(1):128142.

https://www.redalyc.org/pdf/3215/321531779011.pdf

7. Citlalli Hernández, Sara Ochoa, Iliana Yaschine. El programa de comedores comunitarios: análisis de su diseño e instrumentación. 2015.

8. Aguilar-Orejel L. Encuesta directa, Cultura política y participación electoral en elecciones locales de la ciudad de Tepic, Nayarit, Tesis de Maestría en Desarrollo Económico Local, UAN, Tepic, 2010. https://www.eumed.net/librosgratis/2013a/1329/1329.pdf

9. Pérez Lizaur AB. Sistema Mexicano de Alimentos Equivalentes. 2008. Editorial FNS. Mexico.

10. Organización Mundial de la Salud. Dieta, nutrición y prevención de las enfermedades crónicas. Informe de una consulta de expertos conjunta FAO/ OMS. Serie Informes Técnicos OMS 916. Ginebra: OMS, 2003.

11. Bonvecchio-Arenas A, Fernández-Gaxiola AC, Belausteguigoitia MP. Guías alimentarias y de actividad física. Academia nacional de medicina. 2015:24.

12. Organización para la Cooperación y Desarrollo Económicos (OECD). ¿Cómo se compara México?. [Internet]. México; 2017 [Citado el 20 de Oct. de 2018]; Disponible en: https://www.oecd.org/mexico/Health-at-a-Glance-2017Key-Findings-MEXICO-in-Spanish.pdf.

13. Li X, Shimizu Y, Kmura I. Gut microbial metabolite shortchain fatty acids and obesity. Biosci Microbiota, Food Heal. 2017;36(4):135-140. https://doi.org/10.12938/bmfh.17010

14. Latham M. Nutrición humana en el mundo en desarrollo. 2002. Roma, Italia: fao. http://www.fao.org/3/w0073s/w0073s00.htm

15. Banu C. Suveranitate. Securitate si siguranta alimentara, Organización de las Naciones Unidas para la Agricultura y la Alimentación (Sovereignty, Security and Food Safety). Bucureşti. ASAB Press, 2007.

16. Misra V, Shrivastava AK, Shukla SP, Ansari MI. Effect of sugar intake towards human health. Saudi J. 2016;1(2):29-36. http://scholarsmepub.com/wpcontent/uploads/2016/09/SJM-1229-36.pdf

17. Neacsu NA. Effects of carbohydrate consumption. Case of Study: Carbohydrates in bread. Bull of the Transilvania University of Brasov 2014;7(56):39-44.
http://webbut.unitbv.ro/BU2014/Series\%20V/BULETIN\% 20V/I-05_NEACSU.pdf

18. Seidelmann SB, Claggett B, Cheng S, Henglin M, Shah A, Steffen L, Folsom AR, Rimm EB, Willett WC, Solomon SD. Dietary carbohydrate intake and mortality: a prospective cohort and meta-analysis. Lancet Public Health. 2018; 3(9):419-428.

https://www.thelancet.com/pdfs/journals/lanpub/PIIS246 8-2667(18)30135-X.pdf

19. Dahl WL, Stewart ML (2015). Position of the Academy of Nutrition and Dietetics : Health Implications of Dietary Fiber. 2015. Journal of the Academy of Nutrition and Dietetics. https://doi.org/10.1016/j.jand.2015.09.003

20. Holub I, Gostner A, Theis S, Nosek L, Kudlich T, Melcher R. Novel findings on the metabolic effects of the low glycaemic carbohydrate isomaltulose ( Palatinose e ). British Journal of Nutrition, 2010; 25:1730-1737. https://doi.org/10.1017/S0007114509993874

21. Augustin L. S., Committee S., Kendall C. W. C., Committee S., Jenkins D. J. A., Willett W. C., ... Liu S. Glycemic Index, Glycemic Load and Glycemic Response: an International Scientific Consensus Summit. Nutrition, Metabolism and Cardiovascular Diseases. 2015; 43 (9): 795-815. https://doi.org/10.1016/j.numecd.2015.05.005

22. Yao C. K., Muir J. G., \& Gibson P. R. Alimentary Pharmacology and Therapeutics Review article: insights into colonic protein fermentation, its modulation and potential health implications, (November), Aliment Pharmacol Ther. 2016; 43(2):181-196. https://doi.org/10.1111/apt.13456

23. Gilbert MS, Ijssennagger N, Kies AK, van Mill SWC. Protein fermentation in the gut; implications for intestinal dysfunction in humans, pigs and poultry. Am J Physiol Gastrointest Liver Physiol. 2018; 315(2):159-170. https://doi.org/10.1152/ajpgi.00319.2017

24. Martínez-Jasso I, Becerra-Villezca PA. La alimentacion en Mexico. Un estudio a partir de la Encuesta Nacional de Ingresos y Gastos de los Hogares. Rev Inf y Análisis. 2003: 21:26-37.

25. Moron C, Alonso L, Crovetto M. FAO. Organización de las Naciones Unidas para la Agricultura y la Alimentación (FAO). Oficina regional de la FAO para América Latina y el Caribe. 2005. Cambios en la estructura del consumo de alimentos y nutrientes de América Latina 1979-1981 a 1999-2001.

26. Dávila-Torres J, González-Izquierdo JJ, Barrera-Cruz A. Panorama de la obesidad en México Obesity in Mexico. Rev Med Inst Mex Seguro Soc. 2015;53(2):240-249.

27. Rivera JA, Ph D., Gonzalez T, Olaiz G, Sepúlveda J, Barquera S. Nutrition Transition in Mexico and in Other Latin American Countries. July 2004;62:8-12. https://doi.org/10.1111/j.1753-4887.2004.tb00086.x

28. Pérez AM, Hernández JF. Obesidad y desnutrición en México. Boletín Científico De La Escuela Superior Atotonilco De Tula. 2020;7(13):16-18.

29. Casado-Dones MJ, Fraile-Villar MI, Juárez-Bonilla M, MorenoGonzález C, Martín- Rodríguez M. Dietas milagro bajas en hidratos de carbono o carbofóbicas: perspectiva enfermera desde la evidencia. Enferm Clin. 2016; 26(4):243-249. https://doi.org/10.1016/j.enfcli.2016.03.005

30. Heilbronn LK, Noakes M, Clifton PM. The effect of high- and low-glycemic index energy restricted diets on plasma lipid and glucose profiles in type 2 diabetic subjects with varying glycemic control. J Am Coll Nutr. 2002;21(2):120-7. https://pubmed.ncbi.nlm.nih.gov/11999539/

31. García de Lorenzo y Mateos A., Rodríguez-Montes JA. 
Metabolismo en el ayuno y la agresión. Su papel en el desarrollo de la desnutrición relacionada con la enefermedad. Nutrición Hospitalaria. 2013;6(1):1-9. https://www.redalyc.org/articulo.oa?id=309228933001

32. Kantar Worldpanel México. 2018. Destinan los mexicanos $10.7 \%$ en la compra de leche. Redacción Énfasis Alimentación. [Internet]. México; 2017 [Citado el 18 de Oct. de 2018]; Disponible desde: http://www.alimentacion.enfasis.com/notas/77978destinan-los-mexicanos-107-la-compra-leche.

33. EFSA. Scientific Opinion on Dietary Reference Values for carbohydrates and dietary fibre. European Food Safety Authority. 2010;8(3):1-77. https://doi.org/10.2903/j.efsa.2010.1462

34. FDA. Food Labeling: Revision of the Nutrition and Supplement Facts Labels. Food and Drug Administration, 2016; 81(103), 33741-33999..

35. NOM. Secretaria de economia. Norma Oficial Mexicana, 2020;4:4-40.

36. Bourges H., Casanueva E., \& Rosado J. L. Recomendaciones de ingestión de nutrimentos para la población mexicana. Editorial Médica Panamericana. 2005.

37. Secretaría de Salud. ¿Cuánta fibra dietética se debe consumir?. 2016. Recuperado el 5 de febrero de 2019 en: https://www.gob.mx/salud/articulos/cuanta-fibradietetica-se-debe-consumir

38. Li YO, Komarek AR. Dietary fibre basics: health, nutrition, analysis and applications. Fod Qual Saf. 2017; 1:47-59. https://doi.org/10.1093/fqsafe/fyx007

39. Rodríguez-Ojea A. La transición nutricional y las enfermedades cardiovasculares en la década de los noventa. Acta Médica Cuba. 2003;11(1):7. https://www.medigraphic.com/pdfs/actamedica/acm2003/acm031v.pdf

40. Ibarra LS. Review: Transición alimentaria en México. Razón y Palabra. 2016; 20(94):162-179. https://www.redalyc.org/pdf/1995/199547464012.pdf

41. Andreyeva T., Chaloupka F. J., \& Brownell K. D. . Estimating the potential of taxes on sugar-sweetened beverages to reduce consumption and generate revenue. Preventive Medicine, 2011; 52(6); 413-416. https://doi.org/10.1016/j.ypmed.2011.03.013

42. Moreno T. México campeón mundial en consumo de refresco. El boletin del Universal. 2018. [Internet] [Consultado 12 Nov 2018]. Disponible

en: https://www.eluniversal.com.mx/articulo/nacion/sociedad /2017/03/29/mexico-campeon-mundial-en-consumo-derefresco

43. Garwood P. La OMS recomienda aplicar medidas en todo el mundo para reducir el consumo de bebidas azucaradas y sus consecuencias para la salud. 2018.[Internet] [Consultado 12 Nov 2018]. Disponible en: https://www.who.int/es/newsroom/detail/11-10-2016-who-urges-global-action-tocurtail-consumption-and-health-impacts-of-sugary-drinks

44. OMS. Ingesta de azúcares para adultos y niños. 2015.

45. Instituto Nacional de Estadística, Geografía en Informática (INEGI). Directorio Estadístico Nacional de Unidades Económicas (DENUE) 2011.

46. Interact $\mathrm{T}$. Consumption of sweet beverages and type 2 diabetes incidence in European adults : results from EPICInterAct. Diabetologia. 2013;56:1520-1530. https://doi.org/10.1007/s00125-013-2899-8

47. Koning L. De Malik VS, Rimm EB, Willett WC, Hu FB. Sugarsweetened and artificially sweetened beverage consumption and risk of type 2 diabetes in men $1-3$. The American Journal of Clinical Nutrition. June 2011;93(6):1321-1327. https://doi.org/10.3945/ajcn.110.007922.

48. Vasanti S Malik, Barry M Popkin, George A Bray, Jean-Pierre Després, Walter C Willett, Frank B Hu. Sugar-Sweetened Beverages and Risk of Metabolic Syndrome and Type 2 Diabetes. Diabetes Care. 2010;33:(11). https://doi.org/10.2337/dc10-1079.

49. Duffey KJ, Gordon-larsen P, Steffen LM, Jacobs Jr DR, Popkin BM. Drinking caloric beverages increases the risk of adverse cardiometabolic outcomes in the Coronary Artery Risk Development. Am J Clin Nutr. 2010; 92(4):954-959. https://doi.org/10.3945/ajcn.2010.29478.In

50. Bernstein AM, Koning L, De Flint AJ, Rexrode KM, Willett WC. Soda consumption and the risk of stroke in men and women. Am J Clin Nutr. 2012;95(5):1190-1199. https://doi.org/10.3945/ajcn.111.030205

51. Fung TT, Malik V, Rexrode KM, Manson JE, Willett WC, Hu FB. Sweetened beverage consumption and risk of coronary heart disease in women. The American Journal of Clinical Nutrition. 2009. https://doi.org/10.3945/ajcn.2008.27140

52. V Van Wymelbeke, M-E Béridot-Thérond, V. de L. G. and M. F. Influence of repeated consumption of beverages containing sucrose or intense sweeteners on food intake, Eur J Clin Nutr. 2004; 58:154-161. https://doi.org/10.1038/sj.ejcn.1601762

53. Sámano R, Lilia A, Ventura R, Ytelina E, Martínez G, Rivera B, Ramirez C. Asociación del consumo de bebidas carbonatadas y descalcificación en mujeres en edad reproductiva y no reproductiva de la Ciudad de México Nutrición Hospitalaria, 2013;28(5):1750-1756. https://doi.org/10.3305/nh.2013.28.5.6773

54. Aune D, Chan DSM, Vieira AR, Rosenblatt DAN, Vieira R, Greenwood DC, Norat T. Dietary fructose, carbohydrates, glycemic indices and pancreatic cancer risk: a systematic review and meta-analysis of cohort studies, (April). Analysis of Oncology. 2012;23(10):2536-2546. https://doi.org/10.1093/annonc/mds076.

55. Fuchs MA, Sato K, Niedzwiecki D, Ye X, Saltz LB, Mayer RJ, Messino M. Sugar-Sweetened Beverage Intake and Cancer Recurrence and Survival in CALGB 89803 ( Alliance ). PLOS ONE 2014;9(6). https://doi.org/10.1371/journal.pone.0099816

56. Genkinger JM, Li R, Spiegelman D, Anderson KE, Albanes D, Bergkvist L, Koushik A. Coffee, Tea , and Sugar-Sweetened Carbonated Soft Drink Intake and Pancreatic Cancer Risk : A Pooled Analysis of 14 Cohort Studies, Cancer Epidemiol Biomarkers Prev. 2010;21(2):305-319. https://doi.org/10.1158/1055-9965.EPI-11-0945-T

57. Zhang X, Albanes D, Beeson WL, Brandt PA. Van Den Buring JE, Flood A, Smith-Warner SA. Risk of Colon Cancer and Coffee, Tea, and Sugar-Sweetened Soft Drink Intake : Pooled Analysis of Prospective Cohort Studies, J Natl Cancer Inst. 2010;102(11):771-783. https://doi.org/10.1093/jnci/djq107

58. López-Gonzáñez F, Alarcón-Osuna MA. Cambio generacional del consumo de frutas y verduras en México a través de un análisis de edad-periodo-cohorte 1994-2014. Población y Salud en Mesoamerica. 2018;15(2):23-36. https://doi.org/10.15517/psm.v15i2.28458

59. OMS. Aumentar el consumo de frutas y verduras para reducir el riesgo de enfermedades no transmisibles. 2019. https://www.who.int/elena/titles/fruit_vegetables_ncds/es / 